PROCEEDINGS OF THE

AMERICAN MATHEMATICAL SOCIETY

Volume 133, Number 12, Pages 3551-3560

S 0002-9939(05)07898-6

Article electronically published on June 6, 2005

\title{
A NEW PROPERTY OF A CLASS OF JACOBI POLYNOMIALS
}

\author{
GEORGE CSORDAS, MARIOS CHARALAMBIDES, AND FABIAN WALEFFE \\ (Communicated by Carmen Chicone)
}

\begin{abstract}
Polynomials whose coefficients are successive derivatives of a class of Jacobi polynomials evaluated at $x=1$ are stable. This yields a novel and short proof of the known result that the Bessel polynomials are stable polynomials. Stability-preserving linear operators are discussed. The paper concludes with three open problems involving the distribution of zeros of polynomials.
\end{abstract}

\section{INTRODUCTION}

The new property referred to in the title was observed and conjectured (see Conjecture 1 below) while developing a numerical solution for the Navier-Stokes equations 23. It is related to the fundamental problem of constructing discretization schemes of continuous problems (involving, for example, boundary value problems for various systems of partial differential equations) in such a manner that the associated eigenvalue problems are free of "spurious eigenvalues"; that is, the eigenvalues are all negative. For the purpose of this paper, it is sufficient to consider the problem of constructing polynomial approximations to the eigenvalue problem $d^{2} u / d x^{2}=\lambda u$ for $-1<x<1$ with $u( \pm 1)=0$. It is well known that the solutions to this problem consist of negative eigenvalues $\lambda$ with trigonometric eigenfunctions $u(x)$. If $u_{n}(x)$ is a polynomial approximation of degree $n$ to $u(x)$, then the residual

$$
R_{n}(x):=\lambda u_{n}(x)-\frac{d^{2}}{d x^{2}} u_{n}(x)
$$

is also a polynomial of degree $n$ in $x$ and this relationship can be inverted to obtain

$$
u_{n}(x)=\sum_{k=0}^{[n / 2]} \mu^{k+1} \frac{d^{2 k}}{d x^{2 k}} R_{n}(x),
$$

where $\mu=1 / \lambda$ and $[n / 2]$ denotes the greatest integer less than or equal to $n / 2$. In the Tau method [3, $\S 10.4 .2$ ], the polynomial approximation $u_{n}(x)$ is determined from the boundary conditions $u_{n}( \pm 1)=0$ and the requirement that $R_{n}(x)$ is orthogonal to all polynomials of degree $n-2$ with weight function $W(x) \geq 0$ in the interval $(-1,1)$. Whence for the Jacobi weight function $W_{\alpha, \beta}(x)=(1-x)^{\alpha}(1+x)^{\beta}$, $\alpha, \beta>-1$,

$$
R_{n}(x)=\tau_{0} P_{n}^{(\alpha, \beta)}(x)+\tau_{1} P_{n-1}^{(\alpha, \beta)}(x),
$$

Received by the editors May 28, 2004 and, in revised form, July 9, 2004.

2000 Mathematics Subject Classification. Primary 33C47, 26C10; Secondary 30C15, 33C52.

Key words and phrases. Jacobi and Bessel polynomials, stability, real zeros of polynomials.

(C)2005 American Mathematical Society 
for some $x$-independent coefficients $\tau_{0}$ and $\tau_{1}$, where $P_{n}^{(\alpha, \beta)}(x)$ is the Jacobi polynomial of degree $n$. The Jacobi polynomials are the suitably standardized orthogonal polynomials on the interval $(-1,1)$ with the weight function $W_{\alpha, \beta}(x)$. They have the following beautifully symmetric explicit formula ([7, p. 144], [8, vol. 2, p. 169], [22, p. 68]).

Definition 1. The Jacobi polynomial, $P_{n}^{(\alpha, \beta)}(x)$, of degree $n$, is defined by

$$
P_{n}^{(\alpha, \beta)}(x):=\frac{1}{2^{n}} \sum_{k=0}^{n}\left(\begin{array}{c}
n+\alpha \\
k
\end{array}\right)\left(\begin{array}{c}
n+\beta \\
n-k
\end{array}\right)(x-1)^{n-k}(x+1)^{k}, \quad \alpha, \beta>-1 .
$$

The Jacobi polynomials are generalizations of several families of orthogonal polynomials; to wit, the Chebyshev, Legendre and Gegenbauer (ultraspherical) polynomials (cf. Remark 5). Their importance stems, in part, from the fact that $P_{n}^{(\alpha, \beta)}(x)$ is the only polynomial solution (up to a constant factor, [22, p. 61, Theorem 4.2.2]) of the homogeneous, second-order differential equation

$$
\left(1-x^{2}\right) y^{\prime \prime}+[\beta-\alpha-(\alpha+\beta+2) x] y^{\prime}+n(n+\alpha+\beta+1) y=0 .
$$

In fact, they are the only polynomial solutions of a singular Sturm-Liouville problem on the interval $-1<x<1$, i.e. an eigenvalue problem of the form [3, $\$ 9.2]$

$$
-\left(p(x) y^{\prime}\right)^{\prime}+q(x) y=\lambda w(x) y
$$

with $y^{\prime}( \pm 1)$ bounded, where $p(x)>0, q(x) \geq$ and $w(x) \geq 0$ are continuously differentiable functions on the open interval $(-1,1)$ and $p( \pm 1)=0$. This is directly related to their excellent approximation properties 3 , $\S 9.2 .2, \S 9.6 .1]$.

The Jacobi-Tau approximation to the eigenvalue problem $u^{\prime \prime}=\lambda u, u( \pm 1)=0$ leads to an eigenvalue problem in terms of $\mu, \tau_{0}$ and $\tau_{1}$ (see (2), (3)). These applied considerations have led to the following remarkable conjecture.

Conjecture 1. For every positive integer $n \geq 2$, the polynomial

$$
\varphi_{n}(\mu):=\sum_{k=0}^{[n / 2]}\left(\frac{d^{2 k}}{d x^{2 k}} P_{n}^{(\alpha, \beta)}(x)\right)_{x=1} \mu^{k} \quad(-1<\alpha \leq 1, \beta>-1)
$$

has only real negative zeros, where $P_{n}^{(\alpha, \beta)}(x)$ denotes the Jacobi polynomial of degree $n$.

Remark 1. It follows from (4) that $P_{n}^{(\alpha, \beta)}(-x)=(-1)^{n} P_{n}^{(\beta, \alpha)}(x)$. Thus, by virtue of this symmetry, in (7) (and in the sequel) the derivatives of the Jacobi polynomials may be evaluated at -1 , instead of +1 , subject to the proviso that then the parameters satisfy $-1<\beta \leq 1, \alpha>-1$.

Remark 2. In order to shed light on Conjecture 1 in a concrete setting, we briefly consider the special case when $\alpha=\beta=-\frac{1}{2}$. Then $P_{n}^{\left(-\frac{1}{2},-\frac{1}{2}\right)}(x)=\frac{(2 n) !}{2^{2 n}(n !)^{2}} T_{n}(x)$, where $T_{n}(x)$ denotes the $n^{t h}$ Chebyshev polynomial of the first kind ([22, p. 60]). In terms of powers of $x, T_{n}(x)$ can be written explicitly ([17, p. 24], or [21, p. 37]) as

$$
T_{n}(x)=\frac{n}{2} \sum_{k=0}^{[n / 2]} \frac{(-1)^{k}(n-k-1) !}{(n-2 k) ! k !}(2 x)^{n-2 k} .
$$


Now a calculation, together with an induction argument, shows that (cf. [8, vol. 2, p. $186(26)]$, [21, p. 38, Exercises 1.5.5 and 1.5.6]) for $0 \leq k \leq[n / 2]$,

$$
T_{n}^{(2 k)}(1)=n \frac{2^{2 k}(n+2 k-1) !(2 k) !}{(n-2 k) !(4 k) !} .
$$

Thus, for the Chebyshev polynomials of the first kind, Conjecture 1 asserts that the polynomial

$$
\varphi_{n}(x):=\sum_{k=0}^{[n / 2]} T_{n}^{(2 k)}(1) x^{k}=n \sum_{k=0}^{[n / 2]} \frac{2^{2 k}(n+2 k-1) !(2 k) !}{(n-2 k) !(4 k) !} x^{k}, \quad n \geq 2,
$$

has only real negative zeros. In this case, with the aid of, say, Maple or Mathematica, one can confirm the validity of the conjecture for fairly "large" values of $n$. However, simple examples show that if in (10) the $(2 k)^{t h}$ derivative is replaced by the $k^{\text {th }}$ derivative, then the resulting polynomial need not have only real zeros. Consider, for instance, $\sum_{k=0}^{3} T_{3}^{(k)}(1) x^{k}=1+9 x+24 x^{2}+24 x^{3}$. This polynomial has two non-real zeros. It is also natural to inquire about the validity of Conjecture 1. when the even derivatives of the Chebyshev polynomials are evaluated at a point other than 1. But again we find that such a modification of the polynomial, in (10), need not possess only real zeros. Indeed, set

$$
\varphi_{5}(x, a):=\sum_{k=0}^{[5 / 2]} T_{5}^{(2 k)}(a) x^{k}=a\left(5-20 a^{2}+16 a^{4}+40\left(-3+8 a^{2}\right) x+1920 x^{2}\right)
$$

and

$$
r:=\frac{1}{2} \sqrt{\frac{5}{2}(3-\sqrt{6})}=0.586 \ldots \quad \text { and } \quad R:=\frac{1}{2} \sqrt{\frac{5}{2}(3+\sqrt{6})}=1.845 \ldots
$$

Then an elementary calculation shows that $\varphi_{5}(x, a)$ has two non-real zeros for any real number $a$ such that $|a|<r$ or $|a|>R$. This observation is likely related to the fact that $x= \pm 1$ are the singular points of the corresponding Sturm-Liouville problem (6).

This paper is organized as follows. In Section 2, we use stability analysis (Theorem(1) in conjunction with the Hermite-Biehler Theorem to prove a slightly stronger version of the conjecture (Theorem 21). While there are restrictions on the Jacobi parameters, $\alpha$ and $\beta$, our stability results are also valid for the Chebyshev polynomials (the first and second kind), Legendre polynomials, and a class of Gegenbauer polynomials (Corollary 1). The reality, simplicity and non-negativity of the zeros of certain associated polynomials is established in Theorem 2. We conclude this paper with several corollaries, a new proof of the known result that the Bessel polynomials are stable (Corollary 3), a brief discussion of a class of stability preserving linear operators, and open problems and conjectures (Section 3).

\section{Stability analysis and the proof of Conjecture 1}

A real polynomial, $p(x)$, is said to be a stable polynomial (or a Hurwitz polynomial) if all its zeros lie in the open left half-plane, $\operatorname{Re} z<0$. The importance of stability in analysis and matrix theory are well known (cf. the references in [13]). 
The celebrated Routh-Hurwitz theorem provides a necessary and sufficient condition for a polynomial to be stable (see, for example, [16, §40], [18, §23]). For an elementary derivation of the three basic results in the Routh-Hurwitz theory; namely, the Hermite-Biehler Theorem, the Routh-Hurwitz criterion and the total positivity of a Hurwitz matrix, we refer to 13 . Another frequently used technique involves a continued fraction test for the stability of polynomials (cf. 14 and the references contained therein). However, it seems that none of these familiar results, with the notable exception of the Hermite-Biehler Theorem, provide a tractable stratagem for proving Conjecture 1 even in the special case of the Chebyshev polynomials of the first kind. Our proofs streamline and extend some key ideas introduced by Gottlieb [10] and Gottlieb and Lustman [11.

In the proof of Theorem 1 we will use the following lemmas.

Lemma 1. If $u(x)$ is real and continuously differentiable on $[-1,1]$ with $u(1)=0$ and $W_{\alpha, \beta}(x)=(1-x)^{\alpha}(1+x)^{\beta}$, then

$$
\int_{-1}^{1} \frac{d u^{2}}{d x} W_{\alpha, \beta} d x \leq 0, \quad \text { if } \quad-2<\alpha \leq 0, \beta>0 .
$$

Equality holds only if $u(x)=0$ for all $x$ in $[-1,1]$.

Proof. Integrating by parts,

$$
\int_{-1}^{1} \frac{d u^{2}}{d x} W_{\alpha, \beta} d x=\left[u^{2} W_{\alpha, \beta}\right]_{x=-1}^{x=1}-\int_{-1}^{1} u^{2} \frac{d W_{\alpha, \beta}}{d x} d x .
$$

The first term on the right-hand side vanishes if $\alpha>-2$ and $\beta>0$. The integral on the right-hand side is negative if $\frac{d}{d x} W_{\alpha, \beta}=[\beta-\alpha-(\alpha+\beta) x] W_{\alpha-1, \beta-1} \geq 0$, for $|x| \leq 1$, which is the case if $\alpha \leq 0$ and $\beta>0$.

Lemma 2. If $f_{n}(x)$ is a polynomial of degree $n$ with $f_{n}(1)=0$ and $P_{n}^{(\alpha, \beta)}(x)$ is the Jacobi polynomial of degree $n$ with weight function $W_{\alpha, \beta}(x)=(1-x)^{\alpha}(1+x)^{\beta}$, $\alpha, \beta>-1$, then

$$
\int_{-1}^{1} f_{n} P_{n}^{(\alpha, \beta)} W_{\alpha-1, \beta+1} d x=-\int_{-1}^{1} f_{n} P_{n}^{(\alpha, \beta)} W_{\alpha, \beta} d x .
$$

Proof. Since $f_{n}(x)$ is a polynomial of degree $n$ with $f_{n}(1)=0$, there exist $n$ coefficients $c_{k} \in \mathbb{C}, k=0, \ldots, n-1$, such that

$$
f_{n}(x)=(1-x) \sum_{k=0}^{n-1} c_{k} P_{k}^{(\alpha, \beta)}(x) .
$$

Now $W_{\alpha-1, \beta+1}=\frac{1+x}{1-x} W_{\alpha, \beta}$, thus, using expansion (15), we obtain

$$
\int_{-1}^{1} \frac{1+x}{1-x} f_{n} P_{n}^{(\alpha, \beta)} W_{\alpha, \beta} d x=c_{n-1} \int_{-1}^{1} x P_{n-1}^{(\alpha, \beta)} P_{n}^{(\alpha, \beta)} W_{\alpha, \beta} d x
$$

and

$$
\int_{-1}^{1} f_{n} P_{n}^{(\alpha, \beta)} W_{\alpha, \beta} d x=-c_{n-1} \int_{-1}^{1} x P_{n-1}^{(\alpha, \beta)} P_{n}^{(\alpha, \beta)} W_{\alpha, \beta} d x,
$$

because $P_{n}^{(\alpha, \beta)}(x)$ is orthogonal to all polynomials of degree less than $n$ with respect to the weight function $W_{\alpha, \beta}(x)$. 
Theorem 1. Let $P_{n}^{(\alpha, \beta)}(x)$ denote the Jacobi polynomial of degree $n$, where $n \geq 2$. If $-1<\alpha \leq 1$ and $\beta>-1$, then the zeros of the polynomial

$$
\Phi_{n}(\mu):=\sum_{k=0}^{n}\left(\frac{d^{k}}{d x^{k}} P_{n}^{(\alpha, \beta)}(x)\right)_{x=1} \mu^{k}
$$

lie in the left half-plane; that is, $\Phi_{n}(\mu)$ is a stable polynomial.

Proof. Consider

$$
f_{n}(x):=\sum_{k=0}^{n} \mu^{k} \frac{d^{k}}{d x^{k}} P_{n}^{(\alpha, \beta)}(x),
$$

where $\mu$ is a root of $\Phi_{n}(\mu)$ so that $f_{n}(1)=0$. Note that $\Phi_{n}(0)=P_{n}^{(\alpha, \beta)}(1)$ $=\left(\begin{array}{c}n+\alpha \\ n\end{array}\right) \neq 0$, hence $\mu \neq 0$. It can be readily checked that

$$
\frac{1}{\mu}\left(f_{n}(x)-P_{n}^{(\alpha, \beta)}(x)\right)=\frac{d}{d x} f_{n}(x) .
$$

Then from (19) or (20) it follows that

$$
\int_{-1}^{1} f_{n} P_{n}^{(\alpha, \beta)} W_{\alpha, \beta} d x=\int_{-1}^{1}\left(P_{n}^{(\alpha, \beta)}\right)^{2} W_{\alpha, \beta} d x
$$

because $P_{n}^{(\alpha, \beta)}(x)$ is orthogonal to all polynomials of degree less than $n$ with respect to the weight function $W_{\alpha, \beta}(x)$.

Multiply (20) by $\overline{f_{n}(x)}$, the complex conjugate of $f_{n}(x)$, and then add to this its complex conjugate to obtain

$$
\left(\frac{1}{\mu}+\frac{1}{\bar{\mu}}\right)\left|f_{n}(x)\right|^{2}-\left(\frac{\overline{f_{n}(x)}}{\mu}+\frac{f_{n}(x)}{\bar{\mu}}\right) P_{n}^{(\alpha, \beta)}(x)=\frac{d}{d x}\left|f_{n}(x)\right|^{2} .
$$

Next multiply both sides of (22) by $W_{\alpha-1, \beta+1}(x)=(1-x)^{\alpha-1}(1+x)^{\beta+1}$ and integrate over $(-1,1)$. Using (14) and (21), we obtain

$$
\begin{aligned}
\left(\frac{1}{\mu}+\frac{1}{\bar{\mu}}\right)\left(\int_{-1}^{1}\left|f_{n}(x)\right|^{2} W_{\alpha-1, \beta+1} d x\right. & \left.+\int_{-1}^{1}\left(P_{n}^{(\alpha, \beta)}\right)^{2} W_{\alpha, \beta} d x\right) \\
& =\int_{-1}^{1}\left(\frac{d}{d x}\left|f_{n}(x)\right|^{2}\right) W_{\alpha-1, \beta+1} d x .
\end{aligned}
$$

Both integrals on the left-hand side are positive and, by Lemma 1, the integral on the right-hand side is negative if $-1<\alpha \leq 1, \beta>-1$, whence

$$
\frac{1}{\mu}+\frac{1}{\bar{\mu}}=\frac{2 \operatorname{Re}(\mu)}{|\mu|^{2}}<0
$$

Remark 3. An alternative to the use of Lemma 2 in the proof of Theorem 1 is to employ Gauss quadrature [10, which is exact for polynomials of degree less than $2 n$ ([2, p. 248], 7, p. 33] or [22, p. 47]). After multiplying (22) by $W_{\alpha-1, \beta+1}=$ $(1-x)^{-1}(1+x) W_{\alpha, \beta}(x)$ and since $\frac{1+x}{1-x} \frac{d}{d x}\left|f_{n}(x)\right|^{2}$ is a polynomial of degree $2 n-1$, 
we can use Gauss quadrature with weight function $W_{\alpha, \beta}(x)$ and weights $w_{j}>0$ to obtain

$$
\left(\frac{1}{\mu}+\frac{1}{\bar{\mu}}\right)\left(\sum_{j=1}^{n} w_{j} \frac{1+x_{j}}{1-x_{j}}\left|f_{n}\left(x_{j}\right)\right|^{2}\right)=\int_{-1}^{1}\left(\frac{d}{d x}\left|f_{n}(x)\right|^{2}\right) \frac{1+x}{1-x} W_{\alpha, \beta} d x,
$$

where $x_{j} \in(-1,1), j=1, \ldots, n$, are the zeros of $P_{n}^{(\alpha, \beta)}(x)$.

Remark 4. Equation (20) is the Tau approximation (see the Introduction) to the eigenvalue problem $u^{\prime}=\lambda u$ in $-1<x<1$ with $u(+1)=0$ (which has no solution).

Remark 5. A glance at Theorem 1 shows that, for appropriate choices of the parameters $\alpha$ and $\beta$, it can be formulated in terms of certain classical orthogonal polynomials. Indeed, if $\alpha=\beta=-1 / 2$, then $P_{n}^{\left(-\frac{1}{2},-\frac{1}{2}\right)}(x)=\frac{(2 n) !}{2^{2 n}(n !)^{2}} T_{n}(x)$, where $T_{n}(x)$ denotes the $n^{\text {th }}$ Chebyshev polynomial of the first kind ([22, p. 60], [17, Chapter 1], 21, Chapter 1]). The polynomials $\left\{T_{n}(x)\right\}_{n=0}^{\infty}$ are orthogonal with respect to the weight function $W(x)=(1-x)^{-1 / 2}$ on the interval $(-1,1)$. If $\alpha=\beta=1 / 2$, then $P_{n}^{\left(\frac{1}{2}, \frac{1}{2}\right)}(x)=\frac{(2 n+2) !}{2^{2 n+1}((n+1) !)^{2}} U_{n}(x)$, where $U_{n}(x)$ denotes the $n^{\text {th }}$ Chebyshev polynomial of the second kind ([22, p. 60]). The polynomials $\left\{U_{n}(x)\right\}_{n=0}^{\infty}$ are orthogonal with respect to the weight function $W(x)=(1-x)^{1 / 2}$ on the interval $(-1,1)$. If $\alpha=\beta=0$, then $P_{n}^{(0,0)}(x)=P_{n}(x)$, where $P_{n}(x)$ denotes the $n^{\text {th }}$ Legendre polynomial ([22, p. 60], 20, Chapter 10]). The polynomials $\left\{P_{n}(x)\right\}_{n=0}^{\infty}$ are orthogonal with respect to the weight function $W(x) \equiv 1$ on the interval $(-1,1)$. As a last example, we recall that if $\alpha=\beta=\nu-1 / 2$, where $\nu>-\frac{1}{2}$ and $\nu \neq 0$, (a caveat is in order for the case when $\nu=0$; see [8, vol. 2, p. 174]), then $P_{n}^{\left(\nu-\frac{1}{2}, \nu-\frac{1}{2}\right)}(x)=\frac{\left(\nu+\frac{1}{2}\right)_{n}}{(2 \nu)_{n}} C_{n}^{\nu}(x)$, where $C_{n}^{\nu}(x)$ denotes the $n^{\text {th }}$ Gegenbauer (or ultraspherical) polynomial (20, p. 277]). The polynomials $\left\{C_{n}^{\nu}(x)\right\}_{n=0}^{\infty}$ are orthogonal with respect to the weight function $W(x)=(1-x)^{\nu-1 / 2}$ on the interval $(-1,1)$. Thus, with the above nomenclature, as an immediate consequence of Theorem 1, we obtain the following result.

Corollary 1. Let $p_{n}(x)$ be a real polynomial and set $\Phi_{n}(\mu):=\sum_{k=0}^{n} p_{n}^{(k)}(1) \mu^{k}$. Then the polynomial $\Phi_{n}(\mu)$ is stable in each of the following cases:

(a) $p_{n}(x):=T_{n}(x) \quad$ (Chebyshev polynomial of the first kind);

(b) $p_{n}(x):=U_{n}(x) \quad$ (Chebyshev polynomial of the second kind);

(c) $p_{n}(x):=P_{n}(x) \quad$ (Legendre polynomial);

(d) $p_{n}(x):=C_{n}^{\nu}(x),-1 / 2<\nu \leq 3 / 2, \nu \neq 0 \quad$ (Gegenbauer polynomial).

In the proof of Theorem 2, we will appeal to the following classical version of the Hermite-Biehler Theorem.

The Hermite-Biehler Theorem ([15, p. 305], [18, p. 13], [13]). Let

$$
f(z):=p(z)+i q(z)=: a_{n} \prod_{k=1}^{n}\left(z-z_{k}\right) \quad\left(0 \neq a_{n} \in \mathbb{R}\right),
$$

where $p(z)$ and $q(z)$ are real polynomials of degree $\geq 2$. If $f(z)$ has all its zeros in $\operatorname{Im} z>0$, then $p$ and $q$ have only real, simple zeros which interlace (separate one another) and $d(x):=q^{\prime}(x) p(x)-q(x) p^{\prime}(x)>0$ for all real $x$.

We are now in a position to prove a slightly stronger version of Conjecture 1 
Theorem 2. Let $P_{n}^{(\alpha, \beta)}(x)$ denote the Jacobi polynomial of degree $n$, where $n \geq 2$. If $-1<\alpha \leq 1$ and $\beta>-1$, then the polynomials

$$
\varphi_{n}(\mu):=\sum_{k=0}^{[n / 2]}\left(\frac{d^{2 k}}{d x^{2 k}} P_{n}^{(\alpha, \beta)}(x)\right)_{x=1} \mu^{k}
$$

and

$$
\psi_{n}(\mu):=\sum_{k=0}^{[n / 2]}\left(\frac{d^{2 k+1}}{d x^{2 k+1}} P_{n}^{(\alpha, \beta)}(x)\right)_{x=1} \mu^{k}
$$

have only real, simple negative zeros. Moreover, the zeros of $\varphi_{n}(\mu)$ and $\psi_{n}(\mu)$ interlace.

Proof. By Theorem 1 the zeros of $\Phi_{n}(\mu)$ (see (18) ) lie in the open left half-plane, $\operatorname{Re} \mu<0$. Hence, via the substitution $\mu=i z$, the zeros of $\Phi_{n}(i z)$ lie in the open upper half-plane, $\operatorname{Im} z>0$. Therefore, setting

$$
\begin{aligned}
\Phi_{n}(i z)= & \sum_{k=0}^{[n / 2]}\left(\frac{d^{2 k}}{d x^{2 k}} P_{n}^{(\alpha, \beta)}(x)\right)_{x=1}\left(-z^{2}\right)^{k} \\
& +i z \sum_{k=0}^{[n / 2]}\left(\frac{d^{2 k+1}}{d x^{2 k+1}} P_{n}^{(\alpha, \beta)}(x)\right)_{x=1}\left(-z^{2}\right)^{k} \\
:= & p_{n}(z)+i q_{n}(z),
\end{aligned}
$$

it follows from the Hermite-Biehler Theorem that $p_{n}(z)$ and $q_{n}(z)$ have only real, simple zeros which interlace. Next, set $r_{n}(z):=q_{n}(z) / z$. Then $p_{n}(x)=\varphi_{n}\left(-x^{2}\right)$ and $r_{n}(x)=\psi_{n}\left(-x^{2}\right)$ and whence the zeros of the polynomials $\varphi_{n}(x)$ and $\psi_{n}(x)$ are real, negative and simple. Finally, we infer from the interlacing property of the zeros of the polynomials $p_{n}(x)$ and $q_{n}(x)$, that the zeros of $\varphi_{n}(x)$ and $\psi_{n}(x)$ are also interlacing.

\section{Scholia, StABility PRESERVING OPERATORS AND OPEN PROBlems}

In this section our goal is to deduce some consequences of the above results, provide a new proof that the Bessel polynomials are stable, highlight some lesser known stability preserving operators, and conclude with three open problems.

We first consider a consequence of Theorem 2, Since the polynomials $\varphi_{n}(x)$ and $\psi_{n}(x)$ have real, negative, interlacing zeros and $\left|\operatorname{deg} \varphi_{n}-\operatorname{deg} \psi_{n}\right| \leq 1$, Theorem 2, together with a well-known theorem (see, for example, [15, p. 314], [18, p. 12, Satz $5]$ ), imply the following corollary.

Corollary 2. Let $\varphi_{n}(x)$ and $\psi_{n}(x)$ be the polynomials defined by (27) and (28), respectively. Then for any $a, b \in \mathbb{R}$, the linear combination $a \varphi_{n}(x)+b \psi_{n}(x)$ has only real zeros.

In order to prove our next result, we first recall that the Bessel polynomial of degree $n$ ([9, p. 7 and p. 35], [7, p. 181]) is defined as

$$
y_{n}(x):={ }_{2} F_{0}\left(-n, 1+n ;-;-\frac{x}{2}\right)=\sum_{k=0}^{n} \frac{(n+k) !}{(n-k) ! k !}\left(\frac{x}{2}\right)^{k} .
$$


Now it is known that the zeros of the Bessel polynomials are all simple and lie in the open left half-plane ([9, Theorem 1, p. 74 and Corollary 2, p. 82], 14]). Here we offer the following short proof of this result.

Corollary 3. The Bessel polynomials, $y_{n}(x), n \geq 1$, are stable polynomials.

Proof. Consider the Legendre polynomial, $P_{n}(x)$ (see, for example, [20, p. 157]), where

$$
P_{n}(x):=\frac{1}{2^{n}} \sum_{k=0}^{\left[\frac{n}{2}\right]}(-1)^{k}\left(\begin{array}{l}
n \\
k
\end{array}\right)\left(\begin{array}{c}
2 n-2 k \\
n
\end{array}\right)(x)^{n-2 k} .
$$

Then using a known relation between derivatives of Legendre polynomials and Gegenbauer polynomials ([1, Formulae 22.2.3, 22.5.37], [8, vol. 2, p. 180]), a simple calculation shows that

$$
P_{n}^{(k)}(1) \equiv\left(\frac{d^{k} P_{n}(x)}{d x^{k}}\right)_{x=1}=\frac{(n+k) !}{2^{k}(n-k) ! k !} .
$$

Hence the Bessel polynomial (30) can be written

$$
y_{n}(x) \equiv \sum_{k=0}^{n} P_{n}^{(k)}(1) x^{k},
$$

which is stable by Corollary 1(c).

In stability analysis it is frequently advantageous to consider operations which preserve stability. Thus, for example, it is known that the Hadamard product of two stable polynomials is a stable polynomial [12. Another familiar fact is that the differentiation operator, $D:=d / d x$, is stability preserving, by virtue of the Gauss-Lucas Theorem ([16, p. 12]). In order to cite some other, more general, but useful class of linear operators which preserve stability, we recall the following terminology. A sequence $L=\left\{\gamma_{k}\right\}_{k=0}^{\infty}$ of real numbers is called a multiplier sequence if, whenever the real polynomial $p(x)=\sum_{k=0}^{n} a_{k} x^{k}$ has only real zeros, the polynomial $L[p(x)]:=\sum_{k=0}^{n} \gamma_{k} a_{k} x^{k}$ also has only real zeros. (For a survey of results pertaining to multiplier sequences see [5] and the references contained therein.) For example, if $f(x):=\sum_{k=0}^{\infty} \gamma_{k} \frac{x^{k}}{k !}, \gamma_{k} \geq 0$, is an entire function of order at most one with only real negative zeros, then the Taylor coefficients $\left\{\gamma_{k}\right\}_{k=0}^{\infty}$ form a multiplier sequence ([15, Ch. VIII], [19, p. 115]). In addition, if $p(x)$ is a polynomial having only real negative zeros, then the sequence $L=\{p(k)\}_{k=0}^{\infty}$ is a multiplier sequence by a theorem of Laguerre (cf. [18, Satz 3.2] or [4]).

Theorem 3 ([]). Let $L=\left\{\gamma_{k}\right\}_{k=0}^{\infty}$ be a non-negative multiplier sequence. Then $L[p(z)]$ is a stable polynomial, whenever $p(z)$ is a stable polynomial.

By way of illustration, we note that an immediate consequence of Corollary 1(a), Theorem 2 and Theorem 3 is the following corollary.

Corollary 4. Suppose that $p(x)$ is a polynomial having only real negative zeros. Then the polynomial $\sum_{k=0}^{n} T_{n}^{(k)}(1) p(k) x^{k}$ is stable. Also, the polynomial $\sum_{k=0}^{[n / 2]} T_{n}^{(2 k)}(1) p(k) x^{k}$ has only real negative zeros, where $T_{n}(x)$ denotes the $n^{\text {th }}$ Chebyshev polynomial. 
Similar results hold, mutatis mutandis, for the Chebyshev polynomials of the second kind, Legendre polynomials, Gegenbauer polynomials and the Jacobi polynomials (with parameters $-1<\alpha \leq 1$ and $\beta>-1$ ).

We conclude this paper with the following open problems and conjectures. Conjecture 2 is directly related to the approximation problem discussed in the Introduction.

Conjecture 2. For $\varphi_{n}(\mu)$ as in Theorem 2 and $\forall n \geq 3$, the zeros of the polynomials $\varphi_{n}(\mu)$ and $\varphi_{n-1}(\mu)$ interlace.

Conjecture 3. For $\varphi_{n}(\mu)$ as in Theorem 2 and $\forall n \geq 4$, the zeros of the polynomials $\varphi_{n}(\mu)$ and $\varphi_{n-2}(\mu)$ interlace.

Problem 1. Characterize the linear operators which preserve stability.

If $p(x)$ is a polynomial of degree $n$ with zeros $\left\{\alpha_{k}\right\}_{k=1}^{n}$, where $\operatorname{Re} \alpha_{k}<0, k=$ $1,2, \ldots n$, we define the abscissa of stability of $p(x)$ as $\sigma(p):=\max _{1 \leq k \leq n} \operatorname{Re} \alpha_{k}$. For example, for the Bessel polynomials, $y_{n}(x)$, we have $\sigma\left(y_{n}\right) \leq-2 /[(2 n-3)(2 n-1)]$ ([9, p. 90]).

Problem 2. Consider the stable polynomials

$$
\Phi_{n}(x):=\sum_{k=0}^{n}\left(\frac{d^{k}}{d x^{k}} P_{n}^{(\alpha, \beta)}(x)\right)_{x=1} x^{k},
$$

where $P_{n}^{(\alpha, \beta)}(x)$ is the Jacobi polynomial of degree $n, n \geq 2,-1<\alpha \leq 1$ and $\beta>-1$. Determine the abscissa of stability, $\sigma\left(\Phi_{n}\right)$, for $n=2,3, \ldots$.

Motivated by applied considerations, in this paper we have established the validity of Conjecture 1 for several classes of orthogonal polynomials. Here we call attention to analogous questions involving families of orthogonal polynomials with respect to distributions (measures) of Stieltjes type $d \mu(x)$ (cf. [22, Chapters 1-3]). Modifications of our techniques, in conjunction with the theory of multiplier sequences, may render the following problem tractable.

Problem 3. Characterize the class of all real polynomials, $p_{n}(x)$, of degree $n$, all of whose zeros lie in the interval $(-1,1)$, such that that the associated polynomials $\varphi_{n}(x):=\sum_{k=0}^{\left[\frac{n}{2}\right]} p_{n}^{(2 k)}(1) x^{k}$ possess only real negative zeros.

A solution of Problem 3 could have interesting ramifications in the theory of distribution of zeros of polynomials and transcendental entire functions in the Laguerre-Pólya class (see, for example, [4] or [5]).

\section{REFERENCES}

[1] M. Abramowitz and I. A. Stegun, Handbook of Mathematical Functions, Dover, New York, 1965.

[2] G. E. Andrews, R. Askey and R. Roy, Special Functions, Encyclopedia Math. Appl., vol. 71, Cambridge University Press, Cambridge, 1999. MR.1688958 (2000g:33001)

[3] C. Canuto, M.Y. Hussaini, A. Quarteroni and T.A. Zang, Spectral Methods in Fluid Dynamics, Springer, New York, 1988. MR0917480 (89m:76004)

[4] T. Craven and G. Csordas, Complex zero decreasing sequences, Methods Appl. Anal. 2 (1995), 420-441. MR1376305 (98a:26015)

[5] T. Craven and G. Csordas, Composition theorems, multiplier sequences and complex zero decreasing sequences, Value Distribution Theory and Its Related Topics (G. Barsegian, I. Laine and C. C. Yang, eds.), Kluwer Press (to appear) 
[6] T. Craven and G. Csordas, The Gauss-Lucas theorem and Jensen polynomials, Trans. Amer. Math. Soc. 278 (1983), 415-429. MR0697085 (85d:30031)

[7] T. S. Chihara, An Introduction to Orthogonal Polynomials, Math. Appl., 13, Gordon and Breach Sci. Pub., New York, 1978. MR0481884 (58:1979)

[8] A. Erdélyi et al. Higher Transcendental Functions vols. 1, 2, 3, McGraw-Hill Book Co., New York, 1953. MR0058756 (15:419i)

[9] E. Grosswald, Bessel Polynomials, Lecture Notes in Mathematics, 698, Springer, Berlin, 1978. MR0520397 (80i:33013)

[10] D. Gottlieb, The Stability of Pseudospectral-Chebyshev Methods, Math. Comp. 36 (1981), 107-118. MR 0595045 (82b:65123)

[11] D. Gottlieb and L. Lustman, The spectrum of the Chebyshev collocation operator for the heat equation, SIAM J. Numer. Anal. 20 (1983), 909-921. MR.0714688 (85g:65107)

[12] J. Garloff and D.G. Wagner, Hadamard products of stable polynomials are stable, J. Math. Anal. Appl. 202 (1996), 797-809. MR1408355 (97e:30010)

[13] O. Holtz, Hermite-Biehler, Routh-Hurwitz, and total positivity, Linear Algebra Appl. 372 (2003), 105-110. MR.1999142 (2004f:93092)

[14] R. M. Hovstad, A short proof of a continued fraction test for the stability of polynomials, Proc. Amer. Math. Soc. 105 (1989), 76-79. MR0973839 (90a:30013)

[15] B. Ja. Levin, Distribution of Zeros of Entire Functions, Transl. Math. Mono. 5, Amer. Math. Soc., Providence, RI, 1964; revised ed. 1980. MR0589888 (81k:30011)

[16] M. Marden, Geometry of Polynomials, Math. Surveys no. 3, Amer. Math. Soc. Providence, RI, 1966. MR0225972 (37:1562)

[17] J. C. Mason and D. C. Handscomb, Chebyshev Polynomials, Chapman \& Hall/CRC, New York, 2003. MR1937591 (2004h:33001)

[18] N. Obreschkoff, Verteilung und Berechnung der Nullstellen reeller Polynome, VEB Deutscher Verlag der Wissenschaften, Berlin, 1963. MR0164003 (29:1302)

[19] G. Pólya, Collected Papers, Vol. II Location of Zeros (R. P. Boas, ed.), MIT Press, Cambridge, MA, 1974. MR0505094 (58:21342)

[20] E. Rainville, Special Functions, Chelsea, New York, 1960. MR0107725 (21:6447)

[21] T. J. Rivlin, Chebyshev Polynomials. Approximation Theory to Algebra and Number Theory (2nd ed.), John Wiley \& Sons, Inc., New York, 1990. MR.1060735 (92a:41016)

[22] Szegö, Orthogonal Polynomials, Amer. Math. Soc. Colloq. Pub., vol. XXIII (4th ed.) Amer. Math. Soc., Providence, RI, 1975. MR0372517 (51:8724)

[23] F. Waleffe, Homotopy of exact coherent structures in plane shear flows, Phys. Fluids 15 (2003), 1517-1534. MR 1977897 (2004c:76045)

Department of Mathematics, University of Hawail, Honolulu, Hawail 96822

E-mail address: george@math.hawaii.edu

Department of Mathematics, University of Wisconsin, Madison, Wisconsin 53706

E-mail address: charalam@math.wisc.edu

Department of Mathematics, University of Wisconsin, Madison, Wisconsin 53706

E-mail address: waleffe@math.wisc.edu 